\title{
On the magnetisation and the radiative efficiency of BL Lac jets
}

\author{
E. Sobacchi ${ }^{1,2 \star} \&$ Y. E. Lyubarsky ${ }^{1}$ \\ ${ }^{1}$ Physics Department, Ben-Gurion University, P.O.B. 653, Beer-Sheva 84105, Israel \\ ${ }^{2}$ Department of Natural Sciences, The Open University of Israel, 1 University Road, P.O.B. 808, Raanana 4353701, Israel
}

\begin{abstract}
Theoretical modelling and observations of AGN jets suggest that the non-thermal electrons emitting the observed radiation should (i) carry an amount of energy comparable to the magnetic fields $\left(U_{e} \sim U_{B}\right)$, which is likely the case if the magnetic fields play a dynamically important role in the jet's acceleration process; (ii) cool efficiently in a dynamical time $\left(t_{\text {cool }} \lesssim t_{\text {dyn }}\right)$, which is suggested by the fact that a large fraction of the jet's kinetic energy is promptly converted into radiation. These expectations are at odds with the results of the simplest one-zone Self-Synchro-Compton (SSC) model for the Spectral Energy Distribution (SED) of BL Lacs. Indeed, the model predicts $U_{e} \gg U_{B}$ and $t_{\text {cool }} \gg t_{\text {dyn }}$ for most of the objects. Here we closely investigate one of the key assumptions of this model, namely that the momentum distribution of the non-thermal electrons is isotropic. We find that this assumption may be an oversimplification. If the magnetic energy is dissipated via a turbulent MHD cascade, the highest energy electrons may instead retain a small pitch angle. Since the synchrotron emissivity is suppressed when the pitch angle is small, this effect may importantly affect the modelling of the SED. As an illustrative example, we present an anisotropic model for the electron momentum distribution such that $U_{e} \sim U_{B}$ and $t_{\text {cool }} \lesssim t_{\text {dyn }}$ at the same time. Our model manages to simultaneously solve the two problems with one only more free parameter with respect to the usual isotropic one-zone SSC model.
\end{abstract}

Key words: BL Lacertae objects: general - galaxies: jets - radiation mechanisms: nonthermal - plasmas - MHD - turbulence

\section{INTRODUCTION}

The super massive black holes residing in the centre of galaxies are able to launch jets that reach relativistic velocities. These jets produce remarkably non-thermal spectra. Observations suggest that a significant fraction (typically $\sim 15 \%$ ) of the jet's total energy is promptly radiated (e.g. Nemmen et al. 2012). In order to achieve such a high radiative efficiency, the energy dissipation process must involve the efficient acceleration and the subsequent fast cooling of a population of non-thermal particles.

AGN jets are thought to be powered by the rotational energy of the super massive black hole, which is channeled into the outflowing plasma via electromagnetic stresses (e.g. Blandford 1976 Lovelace 1976; Blandford \& Znajek 1977). In this scenario the energy budget of the jet is initially dominated by the Poynting flux, which is gradually converted into the plasma kinetic energy while the flow is accelerated. Theoretical investigation of the energy dissipation process suggests that in this regime the emitting electrons and the electromagnetic fields may carry comparable amounts of energy (see for example Sironi et al. 2015).

Among blazars (i.e. AGN with the jet pointing towards the observer), BL Lacs are the ideal laboratory to test our theoretical

* E-mail: sobacchi@post.bgu.ac.il understanding of the physics of relativistic jets. These objects are particularly attractive due to their simplicity, since they do not show any emission from the black hole accretion disc, as sometimes is the case for Flat Spectrum Radio Quasars (FSRQ). The Spectral Energy Distribution (SED) of BL Lacs can therefore be simply interpreted as due to Self-Synchro-Compton emission from a population of non-thermal electrons (e.g. Tavecchio et al. 2010).

In order to fit the SED, one has to assume a broken power law for the energy distribution of the electrons. One needs just to specify (i) the number density of the non-thermal electrons; (ii) the Lorentz factor $\gamma_{\mathrm{b}}$ of the electrons at the break; (iii) the size $R$, (iv) the magnetic field $B$, and (v) the Doppler factor $\delta$ of the dissipation region. As discussed by Tavecchio et al. (1998), all the parameters of the model are univocally determined by the observed SED.

Despite the apparent simplicity of this model, it is challenging to give a convincing physical interpretation of the results. The reason for this is twofold (see for example Tavecchio \& Ghisellini 2016): (i) the model predicts that the magnetic fields carry just a tiny fraction of the electrons energy, contrary to the theoretical expectation that the two energies are in an approximate equipartition; (ii) the cooling time of the electrons at the break is much longer than the dynamical time, which imply that the jet is extremely radiatively inefficient and raises the further problem to explain the origin of the break itself. Note that a low radiative efficiency would 
be difficult to reconcile with the observations of Nemmen et al. (2012), who instead found that a significant fraction of the jet's energy is promptly radiated.

It has been suggested that relaxing the assumption of a onezone emission model may help to solve this controversy (see for example Tavecchio \& Ghisellini 2016). Though a two-zone emission model can likely provide $U_{e} \sim U_{B}$ and $t_{\text {cool }} \lesssim t_{\text {dyn }}$ for any individual object, we argue that extending this interpretation to the entire BL Lac sample may require some fine tuning. Indeed, since in BL Lacs the luminosity of the synchrotron peak is typically comparable to the luminosity of the IC peak of the SED (e.g. Tavecchio et al. 2010), one would expect the magnetic energy density $U_{B}$ to be comparable to the radiation energy density $U_{\gamma}$. In any model with $U_{e} \sim U_{B}$, this immediately implies that $U_{e} \sim U_{\gamma}$. In a one-zone model, $U_{e} \sim U_{\gamma}$ is naturally achieved if the electrons efficiently radiate their energy in a dynamical time. However, if $U_{\gamma}$ is due to an external radiation field, as is the case in two-zone emission models, it seems to us that there is not any good a priori reason why $U_{e} \sim U_{\gamma}$.

The tension mentioned above motivates a closer examination of the one-zone SSC emission model that is usually adopted to interpret the SED of BL Lacs. Here we challenge one of the key assumptions of this paradigm, namely that the momentum distribution of the non-thermal electrons is isotropic. We show that, if the magnetic energy is dissipated via a turbulent MHD cascade, the highest energy electrons may retain a small pitch angle, which suppresses their synchrotron emission. Taking this effect into account may importantly affect the modelling of the BL Lac SED. Indeed, as an illustrative example we present an anisotropic model for the electron momentum distribution such that (i) the non-thermal electrons and the magnetic fields carry comparable amounts of energy, and (ii) the electrons at the break efficiently cool in a dynamical time. Our model has just one more free parameter than the standard isotropic model.

The paper is organised as follows. In Section 2 we discuss the reason why the highest energy electrons may retain a small pitch angle. In Section 3 we describe the predictions of our anisotropic model for the electron momentum distribution. In Section 4 we compare these predictions with a model that instead assumes an isotropic momentum distribution. In Section 5 we present our main results. Finally, in Section 6 we summarise our conclusions. Throughout this paper we always work in the frame of the source, or equivalently we assume that the source is at redshift $z=0$.

\section{MOTIVATION FOR AN ANISOTROPIC MODEL}

A crucial point in the hydromagnetic jet launching paradigm is understanding how the magnetic energy is dissipated. Since the physical scale of the jet typically exceeds the Larmor radius of the nonthermal particles by many orders of magnitude, it is natural to assume that the energy is brought down to the dissipation scale by a turbulent MHD cascade. In the following we assume the cascade to be injected at the outer scale $R$ of the dissipation region ${ }^{1}$ In a relativistic, optically thin plasma the photon viscosity is unable to

1 Such a turbulent cascade may be triggered by MHD instabilities in a Poynting-dominated jet. Recent PIC simulations have shown that highly tangled magnetic fields may be formed in the kink-unstable region of the jet, resulting in the dissipation of the magnetic energy and the rapid acceleration of a population of non-thermal particles (e.g. Alves et al. 2018, Nalewajko et al. 2018). damp the cascade, which should then proceed unimpeded down to microscopic scales (see for example Zrake et al.|2018).

\subsection{Dissipation of MHD turbulence leads to longitudinal particle heating}

The most important property of MHD turbulence is its strong anisotropy, with the turbulent eddies becoming strongly elongated in the direction of the background magnetic field at small scales. As first proposed by Goldreich \& Sridhar (1995), in the course of the turbulent cascade the ratio of the longitudinal scale of the eddies, $\lambda_{\|}$, to the Alfvén velocity remains equal to the ratio of the perpendicular scale, $\lambda_{\perp}$, to the turbulent velocity (this condition is known as "critical balance"). From this condition, one finds that $\lambda_{\perp}$ and $\lambda_{\|}$are related by

$$
\lambda_{\perp} / \lambda_{\|} \sim \sqrt{\lambda_{\|} / R}
$$

where $R$ is the outer scale, while the cascade is described by a Kolmogorov spectrum in the perpendicular direction.

Thompson \& Blaes (1998) extended the theory to the extreme relativistic regime, when the plasma inertia is negligible (force-free MHD). They argued that an anisotropic cascade is formed also in this case, and that the dissipation occurs at the scale of the current starvation, i.e. when there are not enough charge carriers in the plasma to maintain the currents associated with the Alfvén waves. As pointed out by Thompson (2006), in this case the dissipation of relativistic MHD turbulence heats the particle in the longitudinal direction, and a particle distribution that is strongly elongated in the direction of the background magnetic field might therefore be expected. Thompson (2006); Thompson \& Gill (2014); Gill \& Thompson (2014) suggested that the rapid variability of the GRB prompt emission may be attributed to this anisotropy.

The statement that in collisionless plasmas the anisotropic MHD turbulence decays by heating/accelerating particles along the background magnetic field is general. Indeed, the dissipation occurs at the wave-particle resonances

$$
\omega-\mathbf{k} \cdot \mathbf{v}=n \Omega_{\mathrm{L}},
$$

where $\omega$ and $\mathbf{k}$ are the frequency and the wavenumber, $\mathbf{v}$ is the particle velocity, $\Omega_{\mathrm{L}} \equiv e B / \gamma m c$ is the particle relativistic Larmor frequency, and $n$ is an integer. The cyclotron resonance condition, $n \neq 0$, is satisfied when the longitudinal scale of the wave packet, $\lambda_{\|}$, is of the order of the particle Larmor radius, $r_{\mathrm{L}} \equiv c / \Omega_{\mathrm{L}}$. Since typically $r_{\mathrm{L}} \ll R$, due to the strong anisotropy of MHD turbulence a particle crosses many wave packets during one Larmor orbit and the energy gain averages out. Hence, wave-particle interactions mediated by the cyclotron resonance can be neglected. It was first noticed by Gruzinov (1998); Quataert (1998); Quataert \& Gruzinov (1999) that in this case the dissipation occurs at the Landau resonance, $n=0$. Since the two physical mechanisms of wave-particle interaction at the $n=0$ resonance are due to (i) the longitudinal electric field of the wave and (ii) the interaction between the effective particle's magnetic moment and the longitudinal magnetic perturbation, one is led to the conclusion that the turbulent energy is primarily dissipated onto the longitudinal particle motion.

It has also been found that the turbulent fluctuations tend to align with one another forming small scale current sheets (e.g. Boldyrev 2006, Beresnyak \& Lazarian 2006, Mason et al. 2006), which could be disrupted via magnetic reconnection thus providing an additional dissipation mechanism (e.g. Boldyrev \& Loureiro 2017, Mallet et al. 2017a b, Loureiro \& Boldyrev 2017). Note that 
the background magnetic field, which is much larger than the reconnecting field and lies in the same plane of the current sheet, plays the role of a guide field. Since the magnetic energy is transferred to the plasma particles at the Landau resonance between the particles and the tearing mode that disrupts the current sheet, also in this case one would expect the particles to be heated in the longitudinal direction.

Even if the perpendicular heating is negligible, in a weakly magnetised plasma the fire-hose instability quickly erases any momentum anisotropy (e.g. Parker 1958, Lerche 1966). However, since the fire-hose instability develops once $P_{\|}-P_{\perp}>B^{2} / 4 \pi$ (where $P_{\|}$and $P_{\perp}$ are the parallel and perpendicular pressure components respectively), one immediately sees that this instability is not effective if the magnetic to plasma energy ratio exceeds $1 / 2$. Moreover, a significant angular spread, say $\left\langle\sin ^{2} \theta\right\rangle \sim 1 / 2$ where $\theta$ is the particle pitch angle, is expected only when the energy ratio drops to very small values. In the following we argue that in the highly magnetised regime (i) some plasma instability may still be able to make the electron momentum distribution isotropic; (ii) such an instability may be ineffective for the most energetic electrons, which concludes our argument.

\subsection{Momentum isotropisation in a highly magnetised plasma by the resonance instability of Alfvén waves}

If the energy of the system was initially stored in the magnetic field, most of the energy release occurs around the equipartition stage when the fire-hose instability does not work. Another isotropisation mechanism is the cyclotron instability that develops at the anomalous cyclotron resonance $(n=-1$ in Eq. 2). In this case, particles with super-Alfvén velocities excite Alfvén waves and at the same time their pitch angle increases, the energy being taken from the longitudinal motion. This process is analogous to the classic cosmic-ray scattering by Alfvén waves (e.g. Lerche 1967, Kulsrud \& Pearce 1968).

In the following we assume the momenta of all the particles to be initially directed along the magnetic field. To fix ideas, let the background magnetic field $B$ be directed in the positive $z$ direction. The Alfvén wave velocity $v_{\mathrm{A}}$ is mildly relativistic at the equipartition stage. In order to determine the stability properties of the plasma, we adapt an argument due to Kulsrud (2005) to the case we are interested in.

\subsubsection{Electron-positron plasma}

We first study the case of an electron-positron plasma. Let us consider a right circularly polarised wave packet with wavelength $\lambda_{\|}$ propagating in the positive $z$ direction. Such a wave is emitted by the positrons at the $n=-1$ resonance, which propagate in the positive $z$ direction, and is absorbed by the electrons at the $n=1$ resonance, which propagate in the negative $z$ direction. Using $n=-1$ in Eq. 22, we may calculate the Lorentz factor of the resonant positrons as

$$
\gamma_{e^{+}, \text {res }} \sim \frac{1}{1-\beta_{\mathrm{A}}} \frac{e B \lambda_{\|}}{m_{e} c^{2}},
$$

where $\beta_{\mathrm{A}} \equiv v_{\mathrm{A}} / c$. In a similar way, the Lorentz factor of the resonant electrons is obtained substituting $n=1$ into Eq. (2), which gives

$$
\gamma_{e^{-}, \text {res }} \sim \frac{1}{1+\beta_{\mathrm{A}}} \frac{e B \lambda_{\|}}{m_{e} c^{2}}
$$

Assuming that the pairs are distributed according to a power law with energy index $\sim-2$, which is ultimately motivated by the observed SED (e.g. Tavecchio et al.2010), one finds the number density of resonant positrons,

$$
n_{e^{+}, \text {res }} \sim \frac{n_{e}}{2 \gamma_{e^{+}, \text {res }}} \sim n_{e} \frac{1-\beta_{\mathrm{A}}}{2} \frac{m_{e} c^{2}}{e B \lambda_{\|}},
$$

and the number density of resonant electrons,

$$
n_{e^{-}, \text {res }} \sim \frac{n_{e}}{2 \gamma_{e^{-}, \text {res }}} \sim n_{e} \frac{1+\beta_{\mathrm{A}}}{2} \frac{m_{e} c^{2}}{e B \lambda_{\|}},
$$

where $n_{e} \sim n_{e^{+}}+n_{e^{-}}$is the total number density of the pairs.

The momentum of the resonant positrons is $p_{e^{+}, \text {res }} \sim$ $\gamma_{e^{+}, \text {res }} m_{e} c$, while the momentum of the resonant electrons is $p_{e^{-} \text {,res }} \sim \gamma_{e^{-} \text {,res }} m_{e} c$. Let $\delta B$ be the amplitude of the wave packet. Since the pitch angle of the positrons diffuses on a time scale $t_{e^{+}, \text {diff }} \sim \Omega_{\mathrm{L}}^{-1}(\delta B / B)^{-2} \sim\left(\gamma_{e^{+}, \text {res }} m_{e} c / e B\right)(\delta B / B)^{-2}$ and the pitch angle of the electrons diffuses on a time scale $t_{e^{-}}$, diff $\sim$ $\left(\gamma_{e^{-}, \text {res }} m_{e} c / e B\right)(\delta B / B)^{-2}$, one finds

$$
\frac{\Delta p_{e^{+}, \mathrm{res}}}{\Delta V \Delta t} \sim \frac{n_{e^{+}, \mathrm{res}} p_{e^{+}, \mathrm{res}}}{t_{e^{+}, \mathrm{diff}}} \sim e B n_{e^{+}, \mathrm{res}}\left(\frac{\delta B}{B}\right)^{2},
$$

and

$$
\frac{\Delta p_{e^{-}, \text {res }}}{\Delta V \Delta t} \sim \frac{n_{e^{-}, \text {res }} p_{e^{-}, \text {res }}}{t_{e^{-}, \text {diff }}} \sim e B n_{e^{-}, \text {res }}\left(\frac{\delta B}{B}\right)^{2} .
$$

These are respectively the momentum density gained (lost) by the wave per unit time due to the resonant interaction with the positrons (electrons). Since $n_{e^{+} \text {,res }}<n_{e^{-} \text {,res }}$, the emission term (7) is smaller than the absorption term (8) and the wave is damped.

Hence, in an electron-positron plasma the instability does not develop and we expect the particle distribution to remain strongly elongated in the direction of the background magnetic field. The synchrotron emissivity depends on the magnetic field through the combination $B \sin \theta$, where $\theta$ is the pitch angle (e.g. Rybicki \& Lightman 1979). Hence, we see that if $\theta \ll 1$ the magnetic field in the dissipation region might be significantly stronger than what it is inferred assuming an isotropic momentum distribution for the nonthermal electrons. Note, however, that the cooling time remains the same even in the limit $\theta \ll 1$.

\subsubsection{Electron-positron-ion plasma}

The presence of an even small (in terms of number density) ion component may completely change the results obtained for an electron-positron plasma. It is important to realise that the amplitude of the right circularly polarised wave considered in the previous section grows due to the resonant interaction with the protons moving in the positive $z$ direction. The fundamental difference with respect to the pair plasma is that there are not negatively charged ions, which would be the analogous of the electron component, that damp the wave. Hence, the amplitude of the wave grows if the number of resonant protons and positrons, which emit the wave, exceeds the number of resonant electrons, which absorb the wave.

In the following we consider the case when the pairs dominate the number density $\left(n_{e} \gg n_{p}\right)$, but the protons dominate the rest mass density of the jet $\left(n_{p} m_{p} \gg n_{e} m_{e}\right)$, which is motivated by a number of independent arguments in the literature (e.g. Sikora \& Madejski 2000, Ghisellini \& Tavecchio 2010). We assume that (i) the pairs are distributed according to a power law with energy index $\sim-2$, and (ii) the proton energy distribution 
is steeper than the electron one, as discussed in more detail below, in which case most of the proton's energy is carried by mildly relativistic particles. Hence, the proton to electron energy density is $U_{p} / U_{e} \sim n_{p} m_{p} / n_{e} m_{e} \log \left(\gamma_{\mathrm{b}}\right)$. Using a typical break Lorentz factor $\gamma_{\mathrm{b}} \sim 10^{4}-10^{6}$ for the non-thermal pairs (e.g. Tavecchio et al. 2010), one finds $U_{p} / U_{e} \sim(100-200) \times n_{p} / n_{e}$. Hence, if $n_{e} \sim(10-100) \times n_{p}$ (e.g. Sikora \& Madejski 2000), the energy carried by the protons does not typically exceed that carried by the pairs by a large factor.

Using the same argument as to derive Eq. (7)-8, one can calculate the momentum density gained by the wave per unit time due to the interaction with the resonant $(n=-1)$ protons, which gives

$$
\frac{\Delta p_{p, \text { res }}}{\Delta V \Delta t} \sim e B n_{p, \text { res }}\left(\frac{\delta B}{B}\right)^{2} .
$$

Combining Eqs. (7)-(9), one sees that the emission is larger than the absorption, and hence the wave grows, if

$$
n_{p, \text { res }}+n_{e^{+}, \text {res }} \gtrsim n_{e^{-}, \text {res }} \text {. }
$$

This is the condition for a particle distribution with all the momenta directed along the background magnetic field to be unstable. If the instability develops, the electrons are isotropised by the absorption of the resonant waves, while the positrons and the protons are isotropised by the emission. In Appendix A we calculate the growth rate of the instability, showing that it is fast enough (with respect, for example, to the dynamical time) for the instability to be indeed effective once the condition $(10)$ is satisfied.

In order to make further progress we need to make some assumptions on the proton energy distribution, which determines $n_{p, \text { res. }}$ First of all, note that if the wavelength is shorter than the proton non-relativistic Larmor radius $\left(\lambda_{\|} \lesssim m_{p} c^{2} / e B\right)$, the number density of the resonant protons equals the total number density of the protons, namely $n_{p \text {,res }} \sim n_{p}$. For longer wavelengths, $n_{p \text {, res }}$ depends on the details of the heating process.

In the following we assume that the protons are distributed according to a power law with energy index $-s$. We take $s>2$, namely we assume that the proton distribution is steeper than the pair distribution. This choice is motivated by the fact that, as a result of the dissipation of non-relativistic MHD turbulence, the proton to electron heating ratio is a decreasing function of the magnetisation, and is already smaller than unity when the thermal and the magnetic energy are in equipartition (e.g. Quataert \& Gruzinov 1999 Howes 2010). However, one should realise that the extrapolation of these results to the relativistic regime is far from obvious, and would require further investigation.

Since the Lorentz factor of the resonant protons is $\gamma_{p \text {,res }} \sim$ $e B \lambda_{\|} / m_{p} c^{2}$, one finds

$$
n_{p, \text { res }} \sim n_{p} \times \begin{cases}1 & \text { if } \quad \lambda_{\|} \lesssim m_{p} c^{2} / e B \\ \left(\frac{m_{p} c^{2}}{e B \lambda_{\|}}\right)^{s-1} & \text { if } \quad \lambda_{\|} \gtrsim m_{p} c^{2} / e B .\end{cases}
$$

The important point is that the number of the resonant protons can exceed the number of resonant pairs $\left(n_{p \text {,res }} \gtrsim n_{e, \text { res }}\right)$ even when the pairs dominate the total number density $\left(n_{p} \ll n_{e}\right)$. The reason for this is the large proton to electron mass ratio, $m_{p} / m_{e} \gg 1$, which implies that the Lorentz factor of the protons resonating with a given $\lambda_{\|}$is significantly smaller than the Lorentz factor of the pairs resonating with the same wave.

Using Eqs. (5) and (6) for $n_{e^{+}, \text {res }}$ and $n_{e^{-}, \text {res }}$, and Eq. (11) for $n_{p, \text { res }}$, we see that the condition (10) is equivalent to

$$
\frac{n_{e} m_{e}}{n_{p} m_{p}} \frac{m_{p} c^{2}}{e B} \lesssim \lambda_{\|} \lesssim\left(\frac{n_{p} m_{p}}{n_{e} m_{e}}\right)^{\frac{1}{s-2}} \frac{m_{p} c^{2}}{e B}
$$

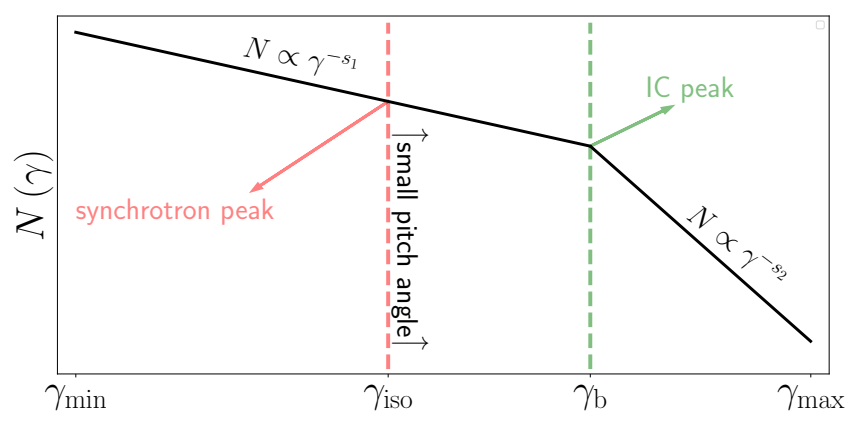

Figure 1. Main features of our model. The energy distribution of the electrons in BL Lac jets is described by a broken power law (see Eq. 14) extending from $\gamma_{\min }$ to $\gamma_{\max }$. The electrons at the break $\left(\gamma \sim \gamma_{\mathrm{b}}\right)$ produce the Inverse Compton peak of the SED. The distribution becomes strongly elongated in the direction of the magnetic field for $\gamma \gtrsim \gamma_{\text {iso. }}$. Since the synchrotron emission by the electrons with $\gamma \gtrsim \gamma_{\text {iso }}$ is suppressed due to their small pitch angles, when $\gamma_{\text {iso }} \lesssim \gamma_{\mathrm{b}}$ the synchrotron peak of the SED is produced by the electrons with $\gamma \sim \gamma_{\text {iso. }}$.

where we have used the fact that $\beta_{\mathrm{A}}$ is of order unity. The Lorentz factor of the pairs resonating with the largest unstable $\lambda_{\|}$can be found from $\gamma_{\text {iso }} m_{e} c^{2} / e B \sim \lambda_{\|}$, which finally gives

$$
\gamma_{\text {iso }} \sim\left(\frac{n_{p} m_{p}}{n_{e} m_{e}}\right)^{\frac{1}{s-2}} \frac{m_{p}}{m_{e}} .
$$

The pairs with $\gamma \lesssim \gamma_{\text {iso }}$ are isotropised due to the resonant interaction with the waves, while those with $\gamma \gtrsim \gamma_{\text {iso }}$ retain a small pitch angle and thus do not radiate by synchrotron.

Since $\gamma_{\text {iso }} \gtrsim m_{p} / m_{e} \sim 2 \times 10^{3}$, it is possible that $\gamma_{\text {iso }} \lesssim \gamma_{\mathrm{b}}$ in a significant fraction of BL Lacs, where the break Lorentz factor can be as large as $10^{5}-10^{6}$ (e.g. Tavecchio et al. 2010). As we discuss in the next section, this fact is important for the modelling of the BL Lac SED. Finally, note that the exact value of $\gamma_{\text {iso }}$ depends on the poorly known details of the particle heating in relativistic MHD turbulence, which determine $s$, and on the composition of the jet.

\section{PREDICTIONS OF THE MODEL}

The main features of our model are sketched in Figure 1 In the following all the physical quantities are defined in the frame of the dissipation region. Following Tavecchio et al. (1998), we assume that the energy distribution of the non-thermal electrons is described by

$$
N(\gamma)=\left\{\begin{array}{lll}
K \gamma^{-s_{1}} & \text { if } & \gamma_{\min }<\gamma<\gamma_{\mathrm{b}} \\
K \gamma_{\mathrm{b}}^{s_{2}-s_{1}} \gamma^{-s_{2}} & \text { if } & \gamma_{\mathrm{b}}<\gamma<\gamma_{\max }
\end{array}\right.
$$

where the scaling constant $K$ has units of $\mathrm{cm}^{-3}$. The spectral indices $s_{1}$ and $s_{2}$ can be determined directly from the SED; one typically finds $s_{1}=1.8-2.2$ and $s_{2}=3.5-5$ (Tavecchio et al. 2010). In the following we adopt a fiducial value $s_{1}=2$.

As discussed in Section 2 we make the further assumption that the momentum of the electrons becomes approximately aligned with the direction of the magnetic field when $\gamma \gtrsim \gamma_{\text {iso }}$

2 In principle, the dependence of the pitch angle on the Lorentz factor of the electrons at $\gamma \sim \gamma_{\text {iso }}$ can be determined by the slope of the SED at frequencies $v \gtrsim v_{\mathrm{s}}$. This would require a detailed fit of the model to the SED of individual objects, which is out of the scope of the paper. 
Since the synchrotron emission is suppressed when the pitch angle is close to zero, if $\gamma_{\text {iso }} \lesssim \gamma_{\mathrm{b}}$ our model predicts the synchrotron radiation to peak at a frequency

$$
v_{\mathrm{s}}=3.7 \times 10^{6} \gamma_{\text {iso }}^{2} B \delta \text {, }
$$

where $\delta$ is the Doppler factor, $\gamma_{\text {iso }}$ is given by Eq. [13, and $v_{\mathrm{s}}$ is measured in Hz. The peak luminosity can be written as

$$
L_{\mathrm{S}}=V \delta^{4} \int N(\gamma) P_{\mathrm{s}}(\gamma) \mathrm{d} \gamma \sim V \delta^{4} N\left(\gamma_{\text {iso }}\right) \gamma_{\text {iso }} P_{\mathrm{S}}\left(\gamma_{\text {iso }}\right)
$$

where $V=4 \pi R^{3} / 3$ and

$$
P_{\mathrm{S}}\left(\gamma_{\text {iso }}\right)=\frac{4}{3} \sigma_{\mathrm{T}} c U_{\mathrm{B}} \gamma_{\text {iso }}^{2},
$$

being $U_{B}=B^{2} / 8 \pi$ the magnetic energy density ${ }^{3}$ Combining Eqs. [16) and 17) we finally get

$$
L_{\mathrm{S}}=\frac{2}{9} \sigma_{\mathrm{T}} c B^{2} R^{3} K \gamma_{\mathrm{iso}} \delta^{4},
$$

where we have used our fiducial $s_{1}=2$. Here $c$ is the speed of light and $\sigma_{\mathrm{T}}$ is the Thompson cross section.

The non-thermal electrons scatter the synchrotron photons to produce the IC peak of the SED. The resulting spectrum depends on the scattering regime of the photons at the synchrotron peak. These photons are scattered in the Thompson regime if

$$
\gamma_{\mathrm{b}} h v_{\mathrm{s}}<\delta m_{e} c^{2}
$$

and in the Klein-Nishina regime otherwise. Here $h$ is the Planck constant and $m_{e}$ is the electron mass. We discuss the two cases separately below.

\subsection{Thompson regime}

The peak of the IC component is produced by the electrons at the break scattering the photons at the synchrotron peak. The peak frequency can be calculated as

$$
v_{c}=\frac{4}{3} \gamma_{b}^{2} v_{s}
$$

The peak luminosity can be written as

$$
L_{\mathrm{c}}=V \delta^{4} \int N(\gamma) P_{\mathrm{c}}(\gamma) \mathrm{d} \gamma \sim V \delta^{4} N\left(\gamma_{\mathrm{b}}\right) \gamma_{\mathrm{b}} P_{\mathrm{c}}\left(\gamma_{\mathrm{b}}\right)
$$

Here

$$
P_{\mathrm{c}}\left(\gamma_{\mathrm{b}}\right)=\frac{4}{3} \sigma_{\mathrm{T}} c U_{\gamma} \gamma_{\mathrm{b}}^{2}
$$

being $U_{\gamma}=L_{\mathrm{S}} / 4 \pi R^{2} c \delta^{4}$ the radiation energy density of the synchrotron photons. Combining Eqs. 21 and 22, we finally get

$$
L_{\mathrm{c}}=\frac{4}{9} \sigma_{\mathrm{T}} R K \gamma_{\mathrm{b}} L_{\mathrm{s}}
$$

where we have used our fiducial $s_{1}=2$.

In order to calculate the cooling time, it is important to realise

\footnotetext{
${ }^{3}$ Note that we are assuming the Doppler amplification to be proportional to $\delta^{4}$, which is the appropriate case if the emitting region moves together with the fluid at the same velocity $\mathbf{v}$. If instead the emitting region is stationary and the fluid inside moves with uniform velocity $\mathbf{v}$ (i.e., it turns "on and off" as it enters and leaves the emitting region), the amplification would be proportional to $\delta^{3} / \Gamma_{\text {jet }}$ (e.g. Lind \& Blandford 1985 Sikora et al. 1997).
}

that in our model the radiative losses of the electrons at the break are dominated by the IC. Hence, the cooling time is

$$
t_{\mathrm{cool}}=\frac{\gamma_{\mathrm{b}} m_{e} c^{2}}{P_{\mathrm{c}}\left(\gamma_{\mathrm{b}}\right)} .
$$

The ratio between the cooling time $t_{\text {cool }}$ and the dynamical time $t_{\text {dyn }}=R / c$ can be presented as

$$
\frac{t_{\mathrm{cool}}}{t_{\mathrm{dyn}}}=\frac{3 \pi m_{e} c^{3} R \delta^{4}}{\sigma_{\mathrm{T}} L_{\mathrm{s}} \gamma_{\mathrm{b}}} .
$$

\subsection{Klein-Nishina regime}

In this case the peak of the IC component is produced by the electrons at the break scattering the photons whose energy equals $m_{e} c^{2}$ in the electron's frame. By construction, the frequency of these photons is below the synchrotron peak. The frequency of the IC peak is then

$$
v_{\mathrm{c}}=\frac{4}{3} \frac{m_{e} c^{2}}{h} \gamma_{\mathrm{b}} \delta
$$

The calculation of $L_{\mathrm{c}}$ and $t_{\text {cool }}$ can be carried out as in the Thompson regime, with the only difference that only the photons with frequency smaller than $\delta m_{e} c^{2} / h \gamma_{\mathrm{b}}=3 \mathrm{v}_{\mathrm{c}} / 4 \gamma_{\mathrm{b}}^{2}$ contribute to the effective $U_{\gamma}$. Since this suppresses $P_{\mathrm{c}}$ by a factor of $\left(3 v_{\mathrm{c}} / 4 \gamma_{\mathrm{b}}^{2} v_{\mathrm{s}}\right)^{1 / 2}$, one finds

$$
L_{\mathrm{c}}=\frac{4}{9} \sigma_{\mathrm{T}} R K L_{\mathrm{s}}\left(\frac{3 v_{\mathrm{c}}}{4 v_{\mathrm{s}}}\right)^{1 / 2}
$$

and

$$
\frac{t_{\mathrm{cool}}}{t_{\mathrm{dyn}}}=\frac{3 \pi m_{e} c^{3} R \delta^{4}}{\sigma_{\mathrm{T}} L_{\mathrm{s}}}\left(\frac{4 v_{\mathrm{s}}}{3 \mathrm{v}_{\mathrm{c}}}\right)^{1 / 2},
$$

which are the analogous of Eqs. 23 and 25 respectively.

\subsection{Final remarks}

The requirement that the observed emission varies on time scales comparable with the dynamical time puts one more constraint to the model, namely

$$
R=c \delta t_{\mathrm{var}} .
$$

where $t_{\mathrm{var}}$ is the observed variability time scale. Substituting $\gamma_{\mathrm{b}}$ from Eq. 20, into Eq. 23, one finds

$$
\sigma_{\mathrm{T}} R K=\frac{9 L_{\mathrm{c}}}{4 L_{\mathrm{s}}}\left(\frac{4 \mathrm{v}_{\mathrm{s}}}{3 \mathrm{v}_{\mathrm{c}}}\right)^{1 / 2} .
$$

It is simple to realise that Eq. 30 is valid also in the Klein-Nishina regime. Note that Eqs. [15, 18, 29, 230 do not contain $\gamma_{\mathrm{b}}$ any more, which allows one to determine $K, R, B, \delta$. Combining Eqs. 20) and 26, and taking into account the condition (19), one eventually finds

$$
\gamma_{\mathrm{b}}=\max \left[\left(\frac{3 v_{\mathrm{c}}}{4 v_{\mathrm{s}}}\right)^{1 / 2},\left(\frac{3 h v_{\mathrm{c}}}{4 \delta m_{e} c^{2}}\right)\right],
$$

which proves that, once $\gamma_{\text {iso }}$ is known, all the free parameters of the model are constrained by observations. 
If the scattering occurs in the Thompson regime, one can calculate $t_{\text {cool }} / t_{\text {dyn }}$ isolating $\gamma_{\mathrm{b}}$ from Eq. 20) and $R$ from Eq. 29, and substituting them into Eq. 25. This finally gives

$$
\frac{t_{\mathrm{cool}}}{t_{\mathrm{dyn}}}=\frac{3 \pi m_{e} c^{4} t_{\mathrm{var}}}{\sigma_{\mathrm{T}} L_{\mathrm{s}}}\left(\frac{4 \mathrm{v}_{\mathrm{s}}}{3 \mathrm{v}_{\mathrm{c}}}\right)^{1 / 2} \delta^{5},
$$

which is valid also in the Klein-Nishina regime.

\section{COMPARISON WITH AN ISOTROPIC MODEL}

\subsection{Predictions of an isotropic model}

The usual one-zone SSC model has five free parameters, which we will name $K_{0}, \gamma_{\mathrm{b} 0}, R_{0}, B_{0}, \delta_{0}$. Since the momentum distribution of the non-thermal electrons is assumed to be isotropic, both the synchrotron and the IC peaks of the SED are produced by the electrons at the break. The frequency and the luminosity of the synchrotron peak are given by

$$
\begin{aligned}
v_{\mathrm{s}} & =3.7 \times 10^{6} \gamma_{\mathrm{b} 0}^{2} B_{0} \delta_{0} \\
L_{\mathrm{s}} & =\frac{2}{9} \sigma_{\mathrm{T}} c B_{0}^{2} R_{0}^{3} K_{0} \gamma_{\mathrm{b} 0} \delta_{0}^{4} .
\end{aligned}
$$

It is simple to realise that Eqs. 29] and (30) are still valid. Hence

$$
\begin{aligned}
R_{0} & =c \delta_{0} t_{\mathrm{var}} \\
\sigma_{\mathrm{T}} R_{0} K_{0} & =\frac{9 L_{\mathrm{c}}}{4 L_{\mathrm{s}}}\left(\frac{4 v_{\mathrm{s}}}{3 v_{\mathrm{c}}}\right)^{1 / 2} .
\end{aligned}
$$

The analogous of Eq. 31 also holds, which finally constrains all the free parameters of the model from the observations.

In order to make a straightforward comparison with our model, here we define $t_{\text {cool, }, 0}$ taking into account the IC cooling only. One finds

$$
\frac{t_{\mathrm{cool}, 0}}{t_{\mathrm{dyn}, 0}}=\frac{3 \pi m_{e} c^{4} t_{\mathrm{var}}}{\sigma_{\mathrm{T}} L_{\mathrm{s}}}\left(\frac{4 v_{\mathrm{s}}}{3 v_{\mathrm{c}}}\right)^{1 / 2} \delta_{0}^{5} .
$$

Note that, since when the momentum distribution is isotropic the synchrotron cooling should be also taken into account, our $t_{\mathrm{cool}, 0}$ is longer than the true cooling time by a factor $1+P_{\mathrm{S}} / P_{\mathrm{c}}=1+L_{\mathrm{S}} / L_{\mathrm{c}}$. Since typically $L_{\mathrm{s}}$ and $L_{\mathrm{c}}$ are of the same order, which can be inferred directly from the SED of individual BL Lacs (e.g. Tavecchio et al. 2010), this correction is a factor of a few.

\subsection{Relation between the physical parameters}

Since the observed quantities (namely $v_{\mathrm{s}}, v_{\mathrm{c}}, L_{\mathrm{s}}, L_{\mathrm{c}}, t_{\mathrm{var}}$ ) are modelindependent by definition, one can find a relation between the physical parameters of our model and those of an isotropic model. Comparing Eq. 15 to Eq. 33 it is simple to realise that

$$
\gamma_{\mathrm{iso}}^{2} B \delta=\gamma_{\mathrm{b} 0}^{2} B_{0} \delta_{0} .
$$

Comparing Eqs. (18) and (34), one finds

$$
B^{2} R^{3} K \gamma_{\text {iso }} \delta^{4}=B_{0}^{2} R_{0}^{3} K_{0} \gamma_{\mathrm{b} 0} \delta_{0}^{4} .
$$

Comparing Eqs. 29] and (35), one finds

$$
R / \delta=R_{0} / \delta_{0}
$$

Finally, comparing Eqs. 30, and 36, one finds

$$
R K=R_{0} K_{0} \text {. }
$$

One can solve Eqs. (38)- 41 in order to express the four parameters $K, R, B, \delta$ as a function of the others, which gives

$$
\begin{aligned}
K & =\left(\frac{\gamma_{\mathrm{b} 0}}{\gamma_{\text {iso }}}\right)^{3 / 4} K_{0} \\
R & =\left(\frac{\gamma_{\text {iso }}}{\gamma_{\mathrm{b} 0}}\right)^{3 / 4} R_{0} \\
B & =\left(\frac{\gamma_{\mathrm{b} 0}}{\gamma_{\text {iso }}}\right)^{11 / 4} B_{0} \\
\delta & =\left(\frac{\gamma_{\text {iso }}}{\gamma_{\mathrm{b} 0}}\right)^{3 / 4} \delta_{0},
\end{aligned}
$$

where $\gamma_{\text {iso }}$ is given by Eq. 13. These expressions give a simple correspondence between the parameters of the two models.

We are now in the position to evaluate how the two models differ in the predicted ratio of (i) the electron to the magnetic energy, and (ii) the cooling to dynamical times. Let $U_{B}$ and $U_{e}$ be the energy density of the magnetic fields and the kinetic energy density of the non-thermal electrons respectively. Since $U_{B}=B^{2} / 8 \pi$, we have $U_{B}=\left(B / B_{0}\right)^{2} U_{B, 0}$. One can calculate $U_{e}=\int \gamma m_{e} c^{2} N(\gamma) \mathrm{d} \gamma \sim$ $K m_{e} c^{2} \log \left(\gamma_{\mathrm{b}} / \gamma_{\min }\right)$, where we have used the distribution (14) with $s_{1}=2$. Neglecting the weak (logarithmic) dependence on $\gamma_{\min }$ and $\gamma_{\mathrm{b}}$, one sees that $U_{e}=\left(K / K_{0}\right) U_{e, 0}$, from which it immediately follows that $U_{e} / U_{B}=\left(B_{0} / B\right)^{2}\left(K / K_{0}\right)\left(U_{e, 0} / U_{B, 0}\right)$. Using Eqs. 42 and 44 we finally get

$$
\frac{U_{e}}{U_{B}}=\left(\frac{\gamma_{\text {iso }}}{\gamma_{\mathrm{b} 0}}\right)^{19 / 4} \frac{U_{e, 0}}{U_{B, 0}} .
$$

Comparing Eqs. 32 and 37, one sees that $t_{\text {cool }} / \delta^{5} t_{\text {dyn }}=$ $t_{\text {cool }, 0} / \delta_{0}^{5} t_{\text {dyn }, 0}$, which using Eq. 45, gives

$$
\frac{t_{\text {cool }}}{t_{\text {dyn }}}=\left(\frac{\gamma_{\text {iso }}}{\gamma_{\mathrm{b} 0}}\right)^{15 / 4} \frac{t_{\text {cool }, 0}}{t_{\text {dyn }, 0}} .
$$

One sees that, in the case $\gamma_{\text {iso }} \lesssim \gamma_{\mathrm{b} 0}$, the ratio of both (i) the electron to the magnetic energy, and (ii) the cooling to the dynamical times predicted by our model can be significantly lower than those predicted by the usual isotropic model.

\section{RESULTS}

Our goal is showing that, assuming that the electron distribution becomes anisotropic at the highest energies, it is possible to have (i) an approximate equipartition between the energy carried by the non-thermal electrons and by the magnetic fields $\left(U_{e} \sim U_{B}\right)$; (ii) the electrons at the break efficiently cooling in a dynamical time $\left(t_{\text {cool }} \lesssim t_{\text {dyn }}\right)$. Combining Eqs. 46) and 47, , we see that

$$
\frac{U_{e}}{U_{B}}\left(\frac{t_{\mathrm{dyn}}}{t_{\mathrm{cool}}}\right)^{19 / 15} \sim \frac{U_{e, 0}}{U_{B, 0}}\left(\frac{t_{\mathrm{dyn}, 0}}{t_{\mathrm{cool}, 0}}\right)^{19 / 15} .
$$

In the following we define the parameter

$$
a \sim \frac{U_{e}}{U_{B}}\left(\frac{t_{\mathrm{dyn}}}{t_{\mathrm{cool}}}\right)^{19 / 15} .
$$

Due to Eq. 48, such a parameter is model-independent (namely, $a \sim a_{0}$ ). If $U_{e} \sim U_{B}$ and $t_{\text {cool }} \lesssim t_{\text {dyn }}$ in the anisotropic model, one would expect $a$ to be distributed above a minimum value of order unity.

In order to calculate the parameter $a$ for individual BL Lacs, we use the results of Tavecchio et al. (2010), who fitted the SED 

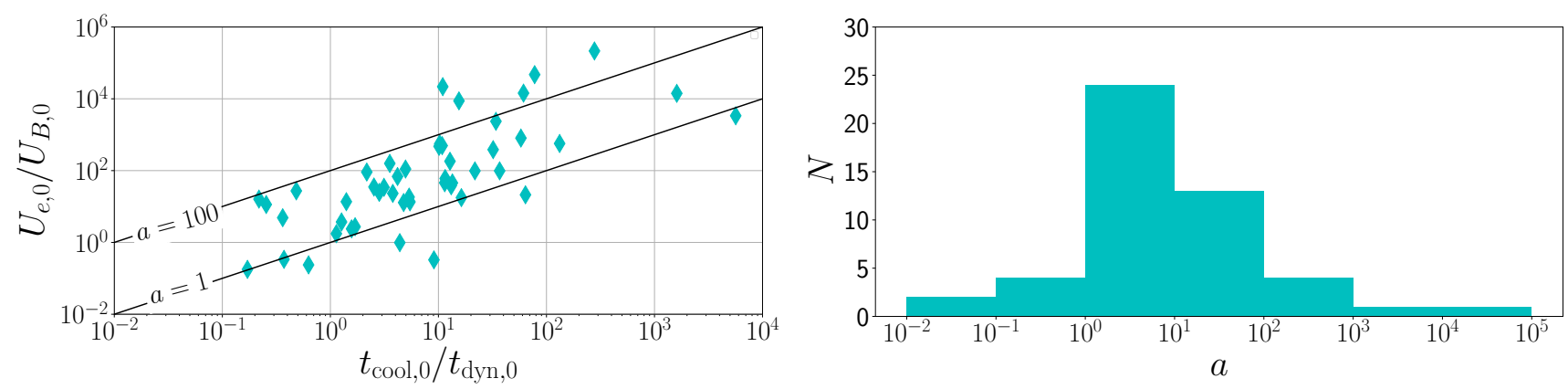

Figure 2. Left panel: ratio of the energy carried by the electrons to the energy carried by the magnetic fields $\left(U_{e, 0} / U_{B, 0}\right)$ versus ratio of the cooling to dynamical times $\left(t_{\mathrm{cool}, 0} / t_{\mathrm{dyn}, 0}\right)$ in a one-zone SSC model that assumes an isotropic electron distribution. Each point represents a BL Lac in the sample of Tavecchio et al. 2010. Right panel: distribution of the model-independent parameter $a \sim\left(U_{e} / U_{B}\right)\left(t_{\mathrm{dyn}} / t_{\mathrm{cool}}\right)^{19 / 15}$ for all the BL Lacs in the sample.

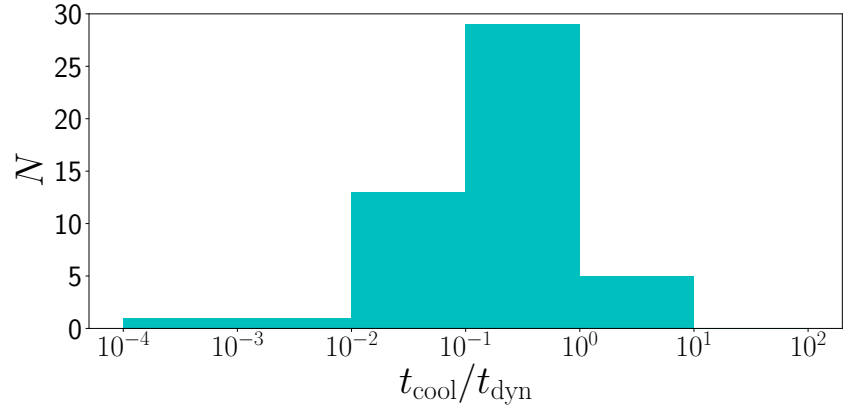

Figure 3. Distribution of the ratio between the cooling to the dynamical times $\left(t_{\mathrm{cool}} / t_{\mathrm{dyn}}\right)$ for all the BL Lacs in the sample. We assume an anisotropic model with energy equipartition between the non-thermal electrons and the magnetic fields $\left(U_{e} \sim U_{B}\right)$.

of a sample of BL Lacs using an isotropic model for the electron distribution. In the left panel of Figure 2 we plot $U_{e, 0} / U_{B, 0}$ versus $t_{\mathrm{cool}, 0} / t_{\mathrm{dyn}, 0}$ for all the BL Lacs in the sample of Tavecchio et al. (2010) ${ }^{4}$ One sees that, while both $U_{e, 0} / U_{B, 0}$ and $t_{\mathrm{cool}, 0} / t_{\mathrm{dyn}, 0}$ have a large scatter and most of the BL Lacs have $U_{e, 0} / U_{B, 0} \gtrsim 1$ and $t_{\text {cool }, 0} / t_{\text {dyn }, 0} \gtrsim 1$, these two quantities are correlated and the combination $a_{0} \sim\left(U_{e, 0} / U_{B, 0}\right)\left(t_{\mathrm{dyn}, 0} / t_{\mathrm{cool}, 0}\right)^{19 / 15}$ is slightly bigger than unity for the majority of the BL Lacs. In the right panel of Figure 2 we show the distribution of $a$ for all the BL Lacs in the sample. Approximately $50 \%$ of the BL Lacs have $1 \lesssim a \lesssim 10$ and $\sim 75 \%$ of them have $1 \lesssim a \lesssim 100$, while only $\sim 12 \%$ of the objects have $a \lesssim 1$. As discussed above, this shows that it is possible to construct an anisotropic model for the electron momentum distribution such that $U_{e} \sim U_{B}$ and $t_{\text {cool }} \lesssim t_{\text {dyn }}$.

\subsection{Proof-of-concept: an anisotropic model with $U_{e} \sim U_{B}$}

As an illustrative example, in the following we calculate all the parameters of our anisotropic model under the assumption that that

\footnotetext{
${ }^{4}$ In order to calculate $t_{\mathrm{cool}, 0}$, (i) we calculate the cooling time due to synchrotron losses only, and (ii) we rescale it by a factor $L_{\mathrm{s}} / L_{\mathrm{c}}$. We infer the luminosities directly from the SED of individual objects.
}

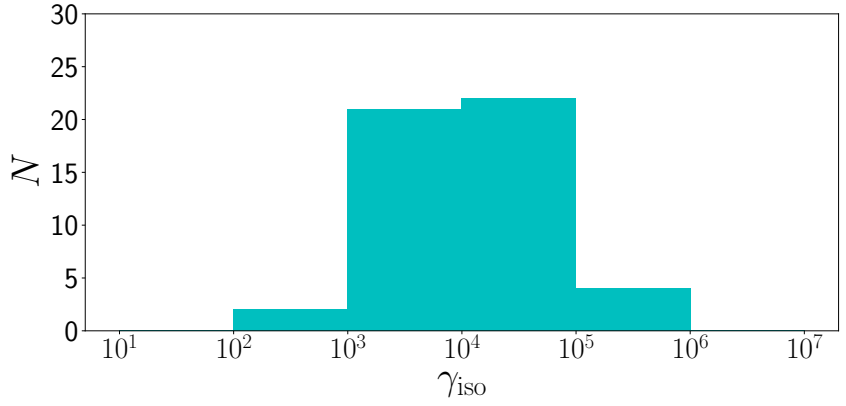

Figure 4. Distribution of $\gamma_{\text {iso }}$ for all the BL Lacs in the sample. We assume an anisotropic model with energy equipartition between the non-thermal electrons and the magnetic fields $\left(U_{e} \sim U_{B}\right)$.

$U_{e} \sim U_{B}{ }^{5}$ In Figure 3 we show the distribution of $t_{\mathrm{cool}} / t_{\mathrm{dyn}}$, which we calculate using Eq. 47) after finding $\gamma_{\text {iso }}$ from Eq. (46. Approximately $60 \%$ of the BL Lacs have $0.1 \lesssim t_{\text {cool }} / t_{\text {dyn }} \lesssim 1$ and $\sim 85 \%$ of them have $0.01 \lesssim t_{\text {cool }} / t_{\text {dyn }} \lesssim 1$, while only $\sim 10 \%$ of the objects have $t_{\text {cool }} / t_{\text {dyn }} \gtrsim 1$. This shows that an anisotropic model with $U_{e} \sim U_{B}$ naturally predicts the electrons at the break to cool efficiently in approximately a dynamical time. Moreover, since the ratio $t_{\text {cool }} / t_{\text {dyn }}$ is typically close to unity, one may speculate that the break in the energy distribution of the non-thermal electrons is separating the electrons whose cooling time is slower/faster than the dynamical time. Therefore the system is self-regulating: electrons are accelerated until they begin to loose the acquired energy. At any $\gamma_{\text {iso }}$, the parameter $\gamma_{\text {iso }} / \gamma_{\mathrm{b}}$, which determines both $U_{e} / U_{B}$ and $t_{\text {cool }} / t_{\text {dyn }}$ is self-adjusted.

In Figure 4 we show the distribution of $\gamma_{\text {iso }}$, namely the Lorentz factor above which the electron distribution becomes elongated in the direction of the magnetic field. As discussed in Section 2. we expect $\gamma_{\text {iso }}$ to be somewhat larger than the proton to electron mass ratio, $m_{p} / m_{e}$. Indeed, only four BL Lacs in the sample require $\gamma_{\text {iso }} \lesssim m_{p} / m_{e}$, while the large majority ( $\sim 84 \%$ ) of the objects have $m_{p} / m_{e} \lesssim \gamma_{\text {iso }} \lesssim 10^{5}$, which is in reasonable agreement with our initial prediction (Eq. 13.

In Figure 5 we show the parameters $K, R, B, \delta$ predicted by our

\footnotetext{
5 Five BL Lacs in the sample of Tavecchio et al. 2010) have $U_{e, 0} \lesssim U_{B, 0}$. For these objects we use the same best fit parameters of the isotropic model adopted by these authors.
} 

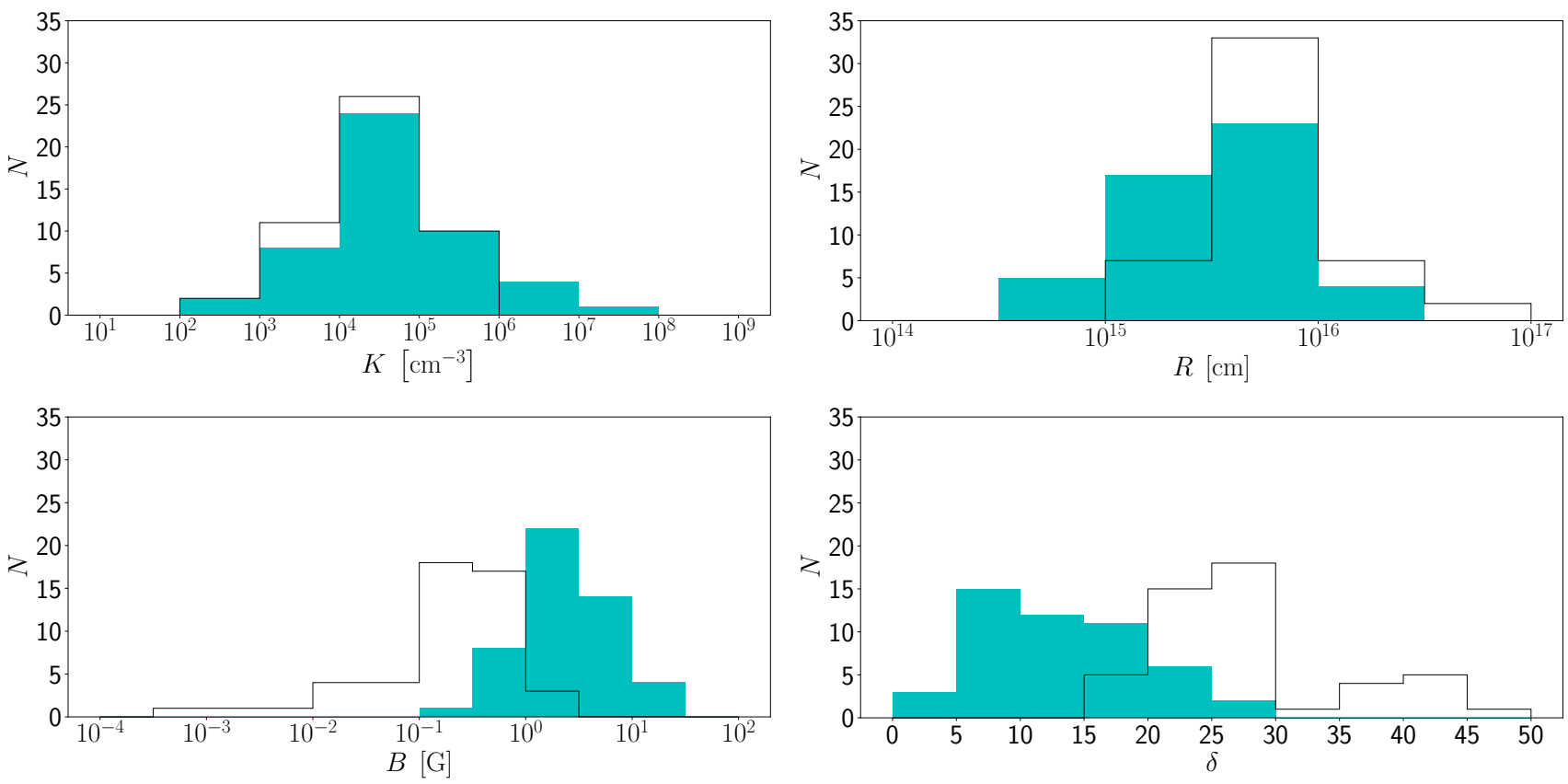

Figure 5. Number density $K$ of the non-thermal electrons (top-left), size $R$ (top-right), magnetic field $B$ (bottom-left) and Doppler factor $\delta$ (bottom-right) of the dissipation region. The cyan histogram corresponds to an anisotropic model with energy equipartition between the non-thermal electrons and the magnetic fields $\left(U_{e} \sim U_{B}\right)$. For comparison, with the thin line we show the distribution that is obtained assuming an isotropic electron distribution.

model, which we find using Eqs. (42)- 45, For comparison, with the thin line we show the distribution that is obtained assuming an isotropic electron distribution. The distribution of the number density of the non-thermal electrons and the distribution of the size of the dissipation region do not change significantly. The distribution of the magnetic field becomes narrower, and the typical field is significantly higher $(\sim 73 \%$ of the BL Lacs have $1 \mathrm{G} \lesssim B \lesssim 10 \mathrm{G})$. Finally, the distribution of the bulk Doppler factor becomes monotonically decreasing, with most of the objects in the range $5 \lesssim \delta \lesssim 25$. Interestingly, even if this was not guaranteed a priori, we find only three objects with $\delta \lesssim 5$ (two of them have $4.5 \lesssim \delta \lesssim 5$ and only one has $\delta \sim 2$ ). This reassures us that our results do not systematically violate the lower limit on $\delta$ that is obtained requiring that the dissipation region is optically thin for pair production $\left(\gamma \gamma \rightarrow e^{+} e^{-}\right.$; e.g. Dondi \& Ghisellini e.g. 1995, 6

\section{CONCLUSIONS}

In this paper we have closely investigated one of the key assumptions that is usually adopted to interpret the SED of blazars, namely that the momentum distribution of the non-thermal electrons emitting the observed radiation is isotropic. We have found that this assumption may be oversimplified. Indeed, if the magnetic energy is dissipated via a turbulent MHD cascade, particles are primarily accelerated along the background magnetic field. In a highly magnetised plasma, the momentum of the lowest energy electrons may be isotropised by resonant wave-particle interactions. However, this

${ }^{6}$ We have checked this constraint not to be violated for any object in the sample of Fan et al. (2014), who calculated the lower limit on the Doppler factor for 457 blazars. Their sample includes $\sim 80 \%$ of the objects in our sample. mechanism is likely inefficient for the highest energy electrons, which may therefore retain a small pitch angle.

Motivated by the physics of energy dissipation in turbulent magnetised plasmas, we have presented a simple anisotropic model where the angular distribution of the electrons momenta depends on the single parameter $\gamma_{\text {iso }}$ : the electron momentum distribution is isotropic if the Lorentz factor is $\gamma \lesssim \gamma_{\text {iso }}$, while the pitch angle

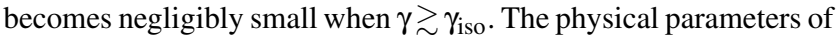
the dissipation region that are derived from the SED modelling are significantly affected by the anisotropy of the electron momentum distribution when $\gamma_{\text {iso }}$ is below the spectral break of the distribution (namely, $\gamma_{\text {iso }} \lesssim \gamma_{\mathrm{b}}$ ), as might be the case in a significant fraction of BL Lacs. The reason for such a difference with respect to the isotropic scenario is that, if $\gamma_{\text {iso }} \lesssim \gamma_{\mathrm{b}}$, the synchrotron peak of the SED is produced by the electrons with $\gamma \sim \gamma_{\text {iso }}$, while the IC peak is produced by the electrons with $\gamma \sim \gamma_{b}$.

We have shown that, with a reasonable choice of the single parameter $\gamma_{\text {iso }}$, it may be possible to construct a one-zone model reproducing the SED of BL Lacs such that (i) the energy carried by the non-thermal electrons and by the magnetic fields are in an approximate equipartition $\left(U_{e} \sim U_{B}\right)$; (ii) the non-thermal electrons efficiently cool in a dynamical time $\left(t_{\text {cool }} \lesssim t_{\text {dyn }}\right)$. As discussed in the introduction, the fact that $U_{e} \sim U_{B}$ and $t_{\text {cool }} \lesssim t_{\text {dyn }}$ is in good agreement with a number of theoretical and observational constraints on AGN jets 7 Our results may therefore help to solve a controversy that was pointed out by Tavecchio \& Ghisellini (2016): indeed, modelling the BL Lac SED with a one-zone Self-

7 Nemmen et al. (2012) found a radiative efficiency of about $15 \%$ for AGN jets. Taking into account that in Poynting dominated jets the fraction of energy going to heat could hardly exceed 50\% (Pe'er 2017), that the electron spectrum is broad, and there are also protons, one concludes that the cooling time at the break, $t_{\text {cool }}$, could not be significantly larger than $t_{\text {dyn }}$. 
Synchro-Compton model that assumes an isotropic momentum distribution for the non-thermal electrons typically gives $U_{e} \gg U_{B}$ and $t_{\mathrm{cool}} \gg t_{\mathrm{dyn}}$. Also note that, since our model predicts the ratio $t_{\text {cool }} / t_{\text {dyn }}$ to be typically close to unity for the electrons at the break of the energy distribution, one may speculate such a break to be associated with the Lorentz factor above which the cooling time becomes shorter than the dynamical time.

The dissipation of the magnetic energy through a turbulent MHD cascade may also explain the rapid variability that is observed in the spectra of blazars. Since the magnetic field in the emitting region is tangled, the radiation in the proper frame is isotropic when averaged over a suitably long time. However, as originally proposed by Thompson (2006) in the context of GRBs, a fast variability on short time scales may be produced due to the fact that the radiation from a locally anisotropic electron distribution is strongly beamed. In blazars, the high energy variability of the spectrum is often explained by "jet in a jet" scenarios that may result from the magnetic reconnection process inside the jet (see for example $\mathrm{Gi}$ annios et al. 2009, 2010, Nalewajko et al.22011). We argue that the emission of highly beamed radiation may instead be the generic product of the energy dissipation in magnetically dominated jets.

Throughout this paper we have mostly been concerned about the statistical properties of BL Lacs. Nevertheless, our model can be used to fit the SED of individual objects. In particular, there are a few objects in the sample of Tavecchio et al. (2010) whose SED is difficult to model assuming an isotropic momentum distribution. As discussed by these authors, the reason is that the fit would require extremely large Doppler factors and small magnetic fields. It would be interesting to see if our model helps to improve the quality of the fit for these objects.

Finally, the fact that the highest energy electrons may retain a small pitch angle is based on a number of assumptions, namely (i) the pairs dominate the total number density $\left(n_{e} \gg n_{p}\right)$, but the protons dominate the total mass density $\left(n_{p} m_{p} \gg n_{e} m_{e}\right)$ of the jet, which is motivated by a number of independent arguments in the literature (e.g. Sikora \& Madejski 2000; Ghisellini \& Tavecchio 2010); (ii) the dissipation of the magnetic energy heats the particles in the direction of the background magnetic field, which is likely the case if the magnetic energy is brought down to the dissipation scale by a turbulent MHD cascade; (iii) the protons are heated less efficiently than the pairs, which is suggested by an analogy with the behaviour of non-relativistic turbulent plasmas. Future studies focusing on the dissipation of the magnetic energy via relativistic MHD turbulence may help to test the correctness of our assumptions (ii) and (iii).

We have not discussed the case of FSRQ yet. In these objects, the strong Compton dominance (which implies that $U_{B} \ll U_{\gamma}$ ) has led different authors to argue that the most promising explanation for the IC peak of the SED is the Comptonization of the radiation provided by a broad-line region or a dusty molecular torus (see for example Sikora et al.2009, Ghisellini et al.|2010). Though the presence of an external photon field makes the detailed modelling of the SED more uncertain than for BL Lacs, it has been suggested that in FSRQ jets (i) the amount of energy carried by the non-thermal electrons is comparable to that carried by the magnetic fields, namely $U_{e} \sim U_{B}$; (ii) the electrons at the break cool efficiently in a dynamical time, namely $t_{\text {cool }} \sim t_{\text {dyn }}$ (e.g. Ghisellini et al. 2010, Ghisellini \& Tavecchio 2015), which would make the interpretation of the model's results less problematic than for BL Lacs. Our model may hardly affect these conclusions in a statistically significant number of FSRQ. The reason is that the typical break Lorentz factor in FSRQ is $\gamma_{b} \sim 10^{2}$ (e.g. Ghisellini et al. 2010), which is smaller than our expected $\gamma_{\text {iso }} \gtrsim 10^{3}$ (see the discussion in Section 2 and in particular Eq. 13. Hence, in FSRQ the electrons at the break may become approximately isotropic, and thus produce both the synchrotron and the IC peaks of the SED.

\section{ACKNOWLEDGEMENTS}

The authors acknowledge useful discussions with Amir Levinson. This research has received funding from the Israeli Science Foundation (grant 719/14) and from the German Israeli Foundation for Scientific Research and Development (grant I-1362-303.7/2016).

\section{REFERENCES}

Alves E. P., Zrake J., Fiuza F., 2018, arXiv:1810.05154

Beresnyak A., Lazarian A., 2006, ApJ, 640, L175

Blandford R. D., 1976, MNRAS, 176, 465

Blandford R. D., Znajek R. L., 1977, MNRAS, 179, 433

Boldyrev S., 2006, Physical Review Letters, 96, 115002

Boldyrev S., Loureiro N. F., 2017, ApJ, 844, 125

Dondi L., Ghisellini G., 1995, MNRAS, 273, 583

Fan J.-H., Bastieri D., Yang J.-H., Liu Y., Hua T.-X., Yuan Y.-H., Wu D.-X., 2014, Research in Astronomy and Astrophysics, 14, 1135

Farmer A. J., Goldreich P., 2004, ApJ, 604, 671

Ghisellini G., Tavecchio F., 2010, MNRAS, 409, L79

Ghisellini G., Tavecchio F., 2015, MNRAS, 448, 1060

Ghisellini G., Tavecchio F., Foschini L., Ghirlanda G., Maraschi L., Celotti A., 2010, MNRAS, 402, 497

Giannios D., Uzdensky D. A., Begelman M. C., 2009, MNRAS, 395, L29

Giannios D., Uzdensky D. A., Begelman M. C., 2010, MNRAS, 402, 1649

Gill R., Thompson C., 2014, ApJ, 796, 81

Goldreich P., Sridhar S., 1995, ApJ, 438, 763

Gruzinov A. V., 1998, ApJ, 501, 787

Howes G. G., 2010, MNRAS, 409, L104

Kulsrud R. M., 2005, Plasma physics for astrophysics. Princeton University Press

Kulsrud R. M., Pearce W., 1968, The Astronomical Journal Supplement, 73, 22

Lerche I., 1966, ApJ, 145, 806

Lerche I., 1967, ApJ, 147, 689

Lind K. R., Blandford R. D., 1985, ApJ, 295, 358

Loureiro N. F., Boldyrev S., 2017, ApJ, 850, 182

Lovelace R. V. E., 1976, Nature, 262, 649

Mallet A., Schekochihin A. A., Chandran B. D. G., 2017a, Journal of Plasma Physics, 83, 905830609

Mallet A., Schekochihin A. A., Chandran B. D. G., 2017b, MNRAS, 468, 4862

Mason J., Cattaneo F., Boldyrev S., 2006, Physical Review Letters, 97, 255002

Nalewajko K., Giannios D., Begelman M. C., Uzdensky D. A., Sikora M., 2011, MNRAS, 413, 333

Nalewajko K., Yuan Y., Chruślińska M., 2018, Journal of Plasma Physics, 84, 755840301

Nemmen R. S., Georganopoulos M., Guiriec S., Meyer E. T., Gehrels N., Sambruna R. M., 2012, Science, 338, 1445

Parker E. N., 1958, Physical Review, 109, 1874

Pe'er A., 2017, ApJ, 850, 200 
Quataert E., 1998, ApJ, 500, 978

Quataert E., Gruzinov A. V., 1999, ApJ, 520, 248

Rybicki G. B., Lightman A. P., 1979, Radiative processes in astrophysics. Wiley, New York

Sikora M., Madejski G., 2000, ApJ, 534, 109

Sikora M., Madejski G., Moderski R., Poutanen J., 1997, ApJ, 484, 108

Sikora M., Stawarz Ł., Moderski R., Nalewajko K., Madejski G. M., 2009, ApJ, 704, 38

Sironi L., Petropoulou M., Giannios D., 2015, MNRAS, 450, 183

Tavecchio F., Ghisellini G., 2016, MNRAS, 456, 2374

Tavecchio F., Ghisellini G., Ghirlanda G., Foschini L., Maraschi L., 2010, MNRAS, 401, 1570

Tavecchio F., Maraschi L., Ghisellini G., 1998, ApJ, 509, 608

Thompson C., 2006, ApJ, 651, 333

Thompson C., Blaes O., 1998, Phys. Rev. D, 57, 3219

Thompson C., Gill R., 2014, ApJ, 791, 46

Zrake J., Beloborodov A. M., Lundman C., 2018, arXiv: 1810.02228

\section{APPENDIX A: CONDITIONS FOR THE RESONANCE INSTABILITY OF ALFVÉN WAVES}

In Section 2 we have studied the stability of a highly magnetised plasma where the momenta of all the particles are aligned with the background magnetic field. Right circularly polarised Alfvén waves are damped due to the absorption by the resonant electrons, while they are emitted by the resonant protons and positrons. Hence, the system is unstable if $n_{p \text {,res }}+n_{e^{+} \text {,res }} \gtrsim n_{e^{-} \text {,res }}$. One can calculate the damping time scale from

$$
\frac{\Delta p_{\text {wave }}}{\Delta V \Delta t} \sim-\frac{\Delta p_{e^{-}, \text {res }}}{\Delta V \Delta t}
$$

where

$$
\frac{\Delta p_{\mathrm{wave}}}{\Delta V \Delta t} \sim-\frac{1}{t_{\mathrm{damp}}} \frac{(\delta B)^{2}}{8 \pi v_{\mathrm{A}}} .
$$

Using Eq. 8, this finally gives

$$
t_{\text {damp }} \sim \frac{B}{8 \pi v_{\mathrm{A}} e n_{e^{-}, \text {res }}} .
$$

In a similar way, one can calculate the growth rate from

$$
\frac{\Delta p_{\text {wave }}}{\Delta V \Delta t} \sim \frac{\Delta p_{e^{+}, \text {res }}}{\Delta V \Delta t}+\frac{\Delta p_{p, \text { res }}}{\Delta V \Delta t},
$$

where

$$
\frac{\Delta p_{\text {wave }}}{\Delta V \Delta t} \sim \frac{1}{t_{\text {growth }}} \frac{(\delta B)^{2}}{8 \pi v_{\mathrm{A}}} .
$$

Using Eqs. (7) and 97, this finally gives

$$
t_{\text {growth }} \sim \frac{B}{8 \pi v_{\mathrm{A}} e\left(n_{p, \text { res }}+n_{e^{+}, \mathrm{res}}\right)} .
$$

One immediately sees that the condition $n_{p \text {,res }}+n_{e^{+} \text {, res }} \gtrsim n_{e^{-} \text {,res }}$ is equivalent to $t_{\text {growth }} \lesssim t_{\text {damp }}$.

The instability is effective if $t_{\text {growth }}$ and $t_{\text {damp }}$ are short with respect to the other relevant time scales of the system. Since the instability develops once $t_{\text {growth }} \lesssim t_{\text {damp }}$, it is sufficient to check $t_{\text {damp }}$ to be short. The wave packet considered above may suffer from the additional damping by the wave-wave interaction with the packets from the turbulent MHD cascade. Following Farmer \& Goldreich (2004), we estimate the time scale for turbulent damping as

$$
t_{\text {turb }} \sim \frac{\sqrt{R \lambda_{\|}}}{v_{\mathrm{A}}},
$$

which is much shorter than the dynamical time in the relevant case $\lambda_{\|} \ll R$. Using Eq. (6) to calculate $n_{e^{-}, \text {res }}$, the ratio $t_{\text {damp }} / t_{\text {turb }}$ may be expressed as

$$
\frac{t_{\mathrm{damp}}}{t_{\mathrm{turb}}} \sim \frac{B^{2}}{8 \pi n_{e} m_{e} c^{2}} \sqrt{\frac{\lambda_{\|}}{R}} .
$$

If the electron and the magnetic energy density are in an approximate equipartition, one sees that $t_{\text {damp }} / t_{\text {turb }} \sim \sqrt{\lambda_{\|} / R} \ll 1$. Hence, $t_{\text {damp }}$ is the relevant time scale of the system, being it of the order of the Larmor time of the resonating electrons.

Finally, note that the particle pitch angle may change due to synchrotron emission, which damps the perpendicular particle motion, and due to IC emission (since the photons are typically scattered exactly in the direction of motion, we argue that IC emission is quite inefficient to produce a diffusion in the pitch angle). The important point is that in our model the cooling time due to both these processes is comparable to the dynamical time (see Figure 3 . Since we have shown the instability discussed in Section 2 to operate on much shorter time scales, we expect that synchrotron and IC processes hardly affect the pitch angle distribution. 